\title{
ASSESSMENT OF EXTERNAL INTERFACE OF AUTONOMOUS VEHICLES
}

\author{
Adam Orlický, Alina Mashko*, Josef Mík \\ Czech Technical University in Prague, Faculty of Transportation Sciences, Department of Vehicle Technology, \\ Horská 3, Prague 2, 128 00, Czech Republic \\ * corresponding author: mashkali@fd.cvut.cz
}

Abstract. The paper deals with the problem of a communication interface between autonomous vehicles $(\mathrm{AV})$ and pedestrians. The introduced methodology for assessing new and existing e-HMI (external HMI) contributes to traffic safety in cities. The methodology is implemented in a pilot experiment with a scenario designed in virtual reality (VR). The simulated scene represents an urban zebra crossing with an approaching autonomous vehicle. The projection is implemented with the help of a head-up display - a headset with a built-in eye tracker. The suggested methodology analyses the pedestrian's decision making based on the visual cues - the signals displayed on the autonomous vehicle. Furthermore, the decision making is correlated to subjects' eye behaviour, based on gaze-direction data. The method presented in this paper contributes to the safety of a vehicle-pedestrian communication of autonomous vehicles and is a part of a research that shall further contribute to the design and assessment of external communication interfaces of AV in general.

KEYWORDS: Autonomous vehicles, eye tracking, graphical user interface, human-machine interaction.

\section{INTRODUCTION}

Autonomous driving eliminates a human agent (the driver), which makes the external communication solely a human-to-car interaction. The transition to autonomous vehicles has been happening gradually, with different levels of automation being set (see the classification of driving automation defined by Society of Automotive Engineers - SAE) [1], where a broader set of assistance systems is available at each of the higher levels of automation. Those systems are to mitigate human errors and their consequences and include not only replacing the driver, but also monitoring their state as well as tracking the environment outside the vehicle (such as traffic, pedestrians and other obstacles). Many modern vehicles are equipped with the systems that place them at a level 3 of automation (by SAE classification), where the set of such systems requires driver intervention (not a full automation) and thus may be deployed on high-class roads. Moreover, at levels of automation from 1 to 4 , numerous driver-car interaction issues arise, e.g., systems' understanding, trust and overtaking [2]. These challenges are to be addressed at higher automation levels (i.e., level 5 and higher). Here, another aspect of the human-machine interaction (HMI) appears a car-pedestrian communication. With pedestrians being a vulnerable traffic group, this aspect is rather important [3]. On non-regulated pedestrian crossings, the pedestrian's decision to walk (cross the road) is often connected with a visual signal from a driver (i.e., a gesture provided by the driver to the pedestrian a nod, or a hand gesture), or a vehicle slow down 4, 5. Such a signal from a driver, or a decision making by a pedestrian, may be perceived differently by the other, which may occasionally lead to unfortunate consequences, i.e., an accident. Moreover, with the arrival of fully autonomous vehicles, where the driver intervention is removed from the system and the decision to slow down is taken by an autonomous vehicle system, this kind of communication shall change. It is, therefore, important to approach the new type of HMI and provide a type of signalling that will be perceived uniformly by human participants.

This research lies in the area of virtual simulations for traffic and contributes to the development of autonomous driving and its communication with humans (pedestrians); the designed method is applicable for testing the reliability of systems with a human agent (external vehicle HMI, in particular) and thus is a part of soft systems engineering field. The method is to be used for designing interfaces for autonomous vehicles and improving their efficiency.

The obtained results are also valuable for the standardization process of HMI of autonomous vehicles. The paper provides an analysis of accidents at pedestrian crossings and an overview of several solutions (external interfaces) for autonomous vehicles to address this problem. Furthermore, we present a methodology for an assessment of such interfaces and its validation via an experiment in a virtual reality scenario, followed by results, analyses and a discussion.

\section{Problem statement}

The following section describes a problem of traffic safety at pedestrian crossings and provides accident statistics. Based on the accident data, analysis of the accident rates and their impact with respect to driver reactions and speed was performed. 


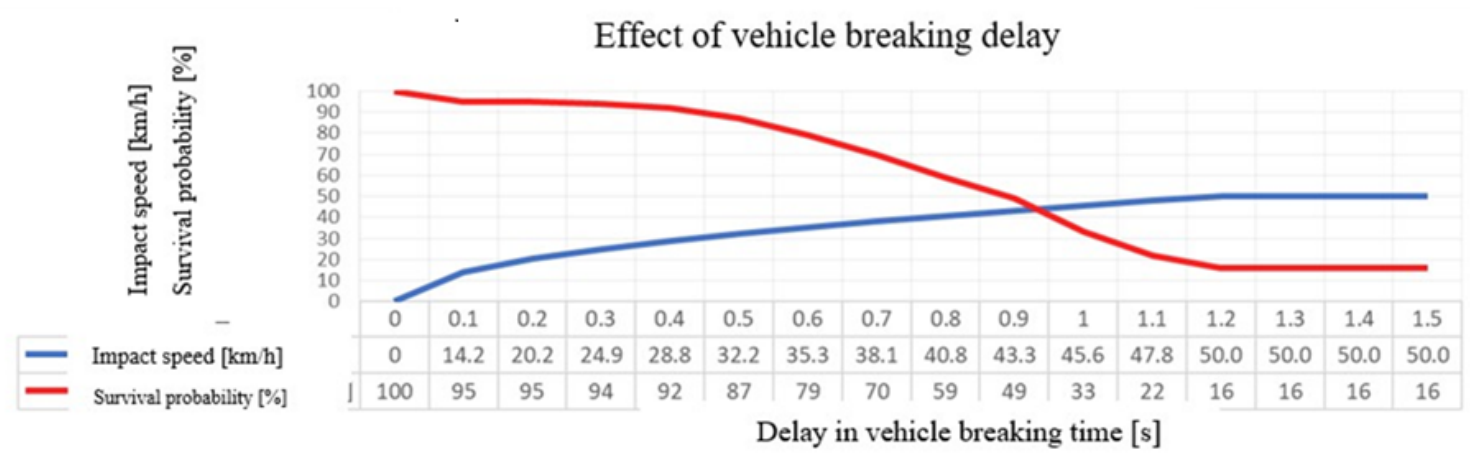

FIGURE 1. Correlation between vehicle braking delay and survival probability.

From the point of view of road safety, pedestrians are not equipped with passive safety tools, which, along with other factors, makes them the most vulnerable traffic participants. According to accident statistics of the Czech Republic (2009-2018), 3500 vehicle-pedestrian accidents occur annually, being $4 \%$ of all registered road accidents. In the year of 2018 , there were 3255 accidents resulting in health damages, among them, 114 were fatal. This gives a probability of $94 \%$ that a pedestrian will get into an accident, i.e., the probability of a fatal outcome is around $4 \%$ [3].

The driver's reaction and the delay in braking has a direct effect on the impact speed, where a braking distance at $50 \mathrm{~km} / \mathrm{h}$ is equal to around $30 \mathrm{~m}$ with no delay. In the case of the $1 \mathrm{~s}$ delay, the impact speed would be $45 \mathrm{~km} / \mathrm{h}$. Based on statistics, at an impact speed of $30 \mathrm{~km} / \mathrm{h}$, the pedestrian survival probability is around $90 \%$, while at the impact speed of $50 \mathrm{~km} / \mathrm{h}$, the probability drops to around $15 \%$. These data are illustrated in Figure 1, which displays the correlation between the braking delay and the survival probability (analyses are based on the data reported in [6] and [7]). Our research investigates the safe distance at which autonomous vehicles need to stop before the pedestrian crossing and shall serve as a basis for a proper and timely signalling and braking at pedestrian crossings.

\section{Available solutions And Related RESEARCH}

Our research was focused on the available concepts of external communication in autonomous vehicles and include related studies in the field as well as an overview of solutions provided by car manufacturers. External interfaces vary in their appearance, design, signalling method and the displayed message. While seeking an original design, vehicle manufacturers are deploying visual interfaces on different body parts. The communication is represented, for example, with animations, text or other LED signals in the zones of lights and windshield. There exists a variety of designs and placements at vehicles by different manufacturers or even the makes of the same manufacturer [8] [10]. At the moment, there is no unified graphical interface design, neither is there a standardized signalling set, or a timing for a displayed message. The development of a single standard shall benefit from a valid interfaceassessing method. This study presents such a method and its verification.

In related experimental designs and methodologies, various kinds of studies approach the problem with behavioural psychology, clinical studies with interviews and questionnaires, observations of a naturalistic behaviour, experimental setups. Variety of methods are well reviewed in [11. We have further narrowed our focus on solely the experimental research. It provides a comparison of a naturalistic study and virtual environment, where each of those have their advantages and disadvantages. In a naturalistic study, a subject gets a real perception of a traffic environment and live experience, which makes them produce natural behaviour. However, a real setup is hard to replicate for every measurement iteration. This complicates providing identical conditions for each measurement, and thus affects the results. Naturalistic measurement data are more challenging to collect. Besides, the investment cost of the live experiment is rather high. This kind of setup is thus recommended for final stages of the design prototype testing. The virtual scenery, on the contrary, is easy to repeat with a high number of subjects and is open to modifications after pilot assessments. The virtual environment allows a precise timing of events and triggers in every iteration and provides cleaner data.

A comparison analysis of both - the live and virtual experiment setup methods - has been performed and is presented in Table 1

The research in a naturalistic environment is more time consuming in data processing, because it involves image processing for live scenarios classification. The results of such studies provide more versatile results with vehicle behaviour. However, the possibility for testing different communication interfaces is limited and has higher costs. Our study suggests a benefit of testing a variety of external communication interface designs with a possibility of testing in particular traffic conditions. Besides that, it also provides the opportunity for acquisition of the physiological behaviour (eye behaviour, in particular) with a perspective of a further development of the user-interface research, 


\begin{tabular}{lcc}
\hline Parameter & VR & Naturalistic \\
\hline Creation of a new scene & Hours/days & Weeks/months \\
Possibility of accidents & Minimal & Medium to High \\
Realness of environment & Low & High \\
Repeatability & High & Low \\
Costs & Low & High \\
Measurement objectiveness & Requires validation & High \\
Feasibility & High & Low \\
\hline
\end{tabular}

TABLE 1. Comparison of testing in virtual reality and naturalistic environment.

e.g., collecting other biometric data, such as heart rate, brain activity etc.

The purpose of this study is to develop a methodology for the research of new e-HMI interfaces. This demands experiment adjustments and multiple replications. The virtual environment meets these requirements from the point of view of operation, financial investments and possibility for a global replication.

\section{The Methodology AND EXPERIMENT SETUP}

The purpose of the suggested methodology is to verify how the external communication on a vehicle affects the pedestrians' perception of a situation and the feeling of safety while crossing the road as compared to a scenario without any external HMI.

The proposed experiment was designed for an e-HMI testing, where a subject's (pedestrian) task is to cross the road when they decide that approaching vehicle gives them the right of way. The experiment used a virtual reality lab setup using HTC Vive Pro Eye for the scenario projection (see Figure 2).

The creation of virtual scenes has a wide implementation in experimental research in the automotive industry. The current method was created within a laboratory that specializes on such a research. Previous projects include a study of human behaviour and HMI research with the help of implementation of simulation technology and VR [12 14.

\subsection{EXPERIMENT SETUP}

The experiment is staged in a city at the spot of a zebra crossing of a two-lane road (one lane in each direction) with a light traffic of autonomous vehicles. The tested vehicle approaches a crossing (from the left-hand side of a subject) and displays the message via e-HMI. The test vehicle's driving speed is $45 \mathrm{~km} / \mathrm{h}$; the space gap between them is $300 \mathrm{~m}$ (four vehicles are tested during one experiment). Cars moving in the opposite direction have the same speed $(45 \mathrm{~km} / \mathrm{h})$ with a time gap of $3 \mathrm{~s}$. No information is displayed on these vehicles and their function is to provide a natural-like traffic scene and to prevent the subject from crossing the road unduly. Thus, the traffic in the opposite direction is only present when there is no tested car

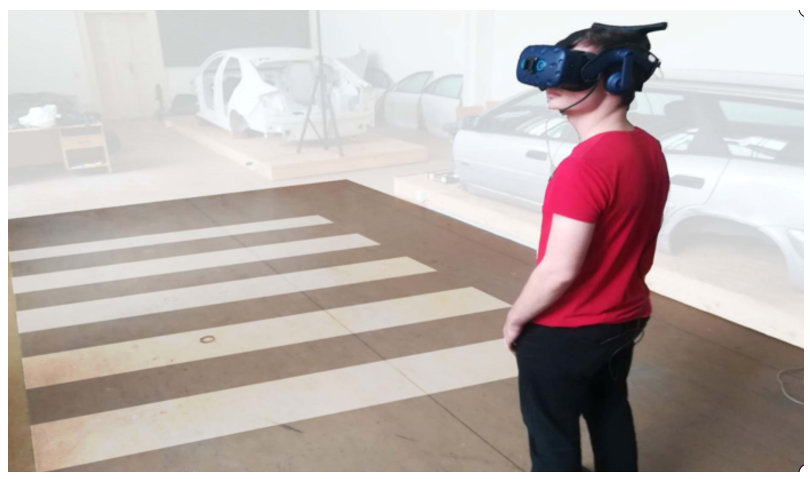

FiguRE 2. Experimental setup in virtual reality lab.

in the vicinity of the pedestrian. The traffic in both lanes is coordinated in such a way that when the tested vehicle arrives at the pedestrian crossing, there is no car in the opposite direction. The algorithm that generates the traffic in the scene schedules the last vehicle in the opposite direction to pass the zebra crossing when the tested car (displaying a signal) is at a distance of $75 \mathrm{~m}$ to the zebra crossing.

This methodology aims to test multiple e-HMI concepts and is designed to prevent the learning factor. This is provided by a randomization algorithm for displaying different types of messages of giving the right of way on the tested vehicles (3 message types have been tested in this experiment), while one of the tested vehicles does not provide any priority (with corresponding information displayed). All subjects are introduced to the signalization types prior to the test and are informed of the meaning of each signalization type.

\subsection{Testing SCEnARIO}

This experiment studies human behaviour as a reaction to vehicle-pedestrian communication and contributes to a higher trust in autonomous vehicles. An external communication interface by Ford was used, where the signals are represented by LED lighting on the top of the windshield [8].

Subjects went through four parts of scenario, where the vehicle is approaching the pedestrian crossing (see the virtual scene on Figure 33. 




FiguRE 3. Experimental virtual scene.

During each of the four vehicle passes, two types of braking behaviour are applied (i.e., normal and aggressive braking). Each braking type is tested with and without an external communication interface. Thus, four vehicle scenarios can be described as follows:

Aggressive braking with a communication interface;

Aggressive braking without a communication interface;

Normal braking with a communication interface;

Normal braking without a communication interface.

Each type of the braking was tested with and without an external communication system, where each of the four vehicle scenarios had a $25 \%$ chance of occurring, this was set to provide a random order of the combinations of the braking behaviour and interface presence.

The types of braking were defined as follows:

In the case of normal braking, the vehicle started to slow down at $50 \mathrm{~m}$, until a full stop at $1 \mathrm{~m}$ from the pedestrian crossing;

In the case of aggressive braking, the vehicle started to slow down at $35 \mathrm{~m}$, until a full stop at $1 \mathrm{~m}$ from the pedestrian crossing.

\subsection{MEASURED DATA}

During the experiment, subjects' eye behaviour was monitored with the help of an eye tracker (part of the HTC Vive Pro Eye used). The head position, rotation, direction of sight and vehicle position were tracked through the whole measurement. Based on these data, the vehicle distance in relation to eye behaviour was monitored, which was consequently taken as a measure of trust to the external communication.
The analyses provide the following output measures:

A. Vehicle distance at the time of the first eye contact The measure represents the first glance at a vehicle (eye fixation on the car body), which stands for the beginning of the pedestrian-car interaction, i.e., the subject starts evaluating the vehicle communication.

B. Vehicle distance at the time when the pedestrian starts crossing the road

The measure represents the distance between the vehicle and the pedestrian crossing at the moment the subject considers it safe to cross the road and starts doing so. (all subjects were instructed to walk when they consider it safe).

C. Entering the pedestrian crossing before the car stops

When the subject enters the pedestrian crossing before the vehicle stops, it is considered they trust the notifications displayed on the vehicle.

\subsection{HARDWARE AND SOFTWARE}

The suggested method deploys an experimental setup in a virtual environment using the head mounted display (HMD) HTC Vive Pro Eye with an integrated eye-tracking module. The virtual scenes were designed in Unity Engine, the virtual objects were directly connected to the gaze behaviour.

\section{Results AND DisCUSSiON}

\subsection{TEST SAMPLE DESCRIPTION}

A total of 18 subjects were tested in the current setup, with the age between 19 and 43 y.o.: (N: 18, min: 19, $\max : 43, \mu$ : $25.8, \sigma^{2}: 49.28$, range: 24$)$.

The subjective assessment included before- and after-test questionnaires to acquire information about 


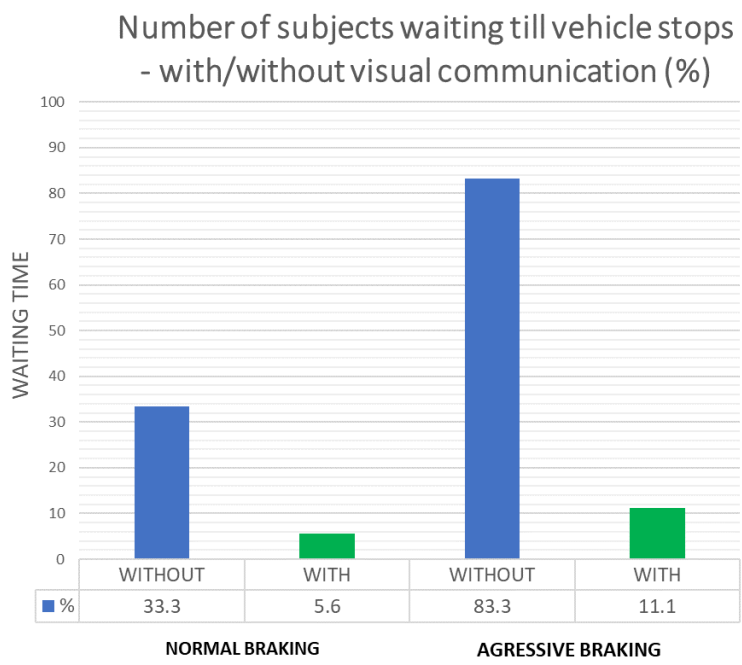

Figure 4. Percentage of subject's waiting time until a full stop of the vehicle.

subjects' behavioural habits of communication to a vehicle or driver while crossing the road as a pedestrian. An after-test questionnaire was related to the perceived experience during the measurement in the virtual reality and subjects' judgement of the tested situation.

All tested subjects reported that they use pedestrian crossing at least twice per day. Minimal crossings are inherent to subjects that commute to and from work and to other activities by a car (active drivers) and suburb residents with minimal extra curriculum activities. Moreover, the active drivers tend to look for a visual contact with the driver when crossing the road, as a part of their pedestrian behaviour.

\subsection{Pedestrian Behaviour During test}

In the case of the aggressive driving (aggressive braking behaviour), the pedestrians' trust in the autonomous vehicle behaviour was the lowest. Per test results, a later deceleration initiation (aggressive braking) resulted in more subjects waiting until the vehicle fully stopped to consider it safe to cross the road in both cases - with or without the visual communication.

The visual communication increased the trust level by decreasing the waiting time. The effect is especially noticeable for the aggressive deceleration mode and makes a positive impact on the waiting time, which decreased by 8 times $(11 \%$ - with the communication as compared to $83 \%$ without the communication). Subjects were able to recognize the visual message at an average distance of $9.6 \mathrm{~m}$ in the aggressive scenario as compared to an average of $15 \mathrm{~m}$ without the visual communication in both scenarios. In the case of the normal braking style, $33 \%$ of subjects waited until the vehicle fully stopped when no visual signalization was present as compared to $5 \%$ with visual signals. Thus, the positive effect of external HMI was noticeable for both driving styles. This effect is displayed in

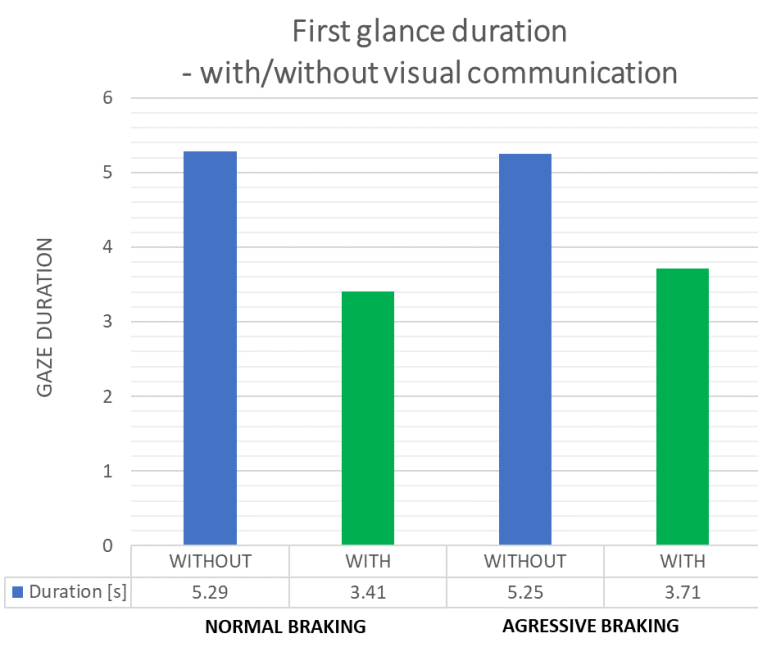

FiguRE 5. Vehicle recognition time per first glance duration.

Figure 4 All except for one subject have entered the crossing sooner when the was vehicle equipped with e-HMI. This is confirmed by the shorter duration of the first glance at a vehicle (Figure 5) that is correlated with a longer vehicle distance from the crossing and at the end of the first glance (see Figure 6, 7). The decision of crossing the road is taken at an average $5.3 \mathrm{~m}$ distance without the visual communication, which decreases with the presence of an external HMI interface

Aggressive - sd: 5.1, median: 7.25, mode: 1.5, min: 1.5, max: 19.4, mean: 8.6 against sd: 0.6 , median: 1.5 , mode: 8.2 , min: 1.5 , max: 3.6 , mean: 1.7 ;

normal - sd: 5.2, median: 12.9 , mode: 8.2 , min: 1.5 , max: 22.4, mean: 13 against sd: 2.4, median: 4.1, mode: 1.5 , min: 1.5 , max: 11 , mean: 3.8 .

\subsection{EYE BEHAVIOUR}

The gaze behaviour didn't significantly differ between the driving modes. However, the presence of eHMI shortened the first fixation duration time in both cases (see Figure 4)

Aggressive - sd: 6.2, median: 7.3, mode: 6.7, min: 1.5, max: 23 against sd:2.2, median: 3.4 , mode: 1.5 , min: 1.5 , max: 9 ;

Normal - sd: 8.02, median: 15.4, mode: 20.7, min: 1.5, max: 33.8 against sd: 3.3 , median: 6.9 , mode: 1.5 , min: 1.5 , max: 11 .

This proves that the presence of an external vehicle communication is efficient as subjects need less time to evaluate the situation.

In the subjective assessment, $61 \%$ of subjects reported they didn't distinguish between different deceleration modes. However, the data shows that $56 \%$ of participants have entered the crossing sooner in the case of normal deceleration. The other $39 \%$ of subjects who recognized the deceleration modes have entered the crossing sooner in the case of normal deceleration. 


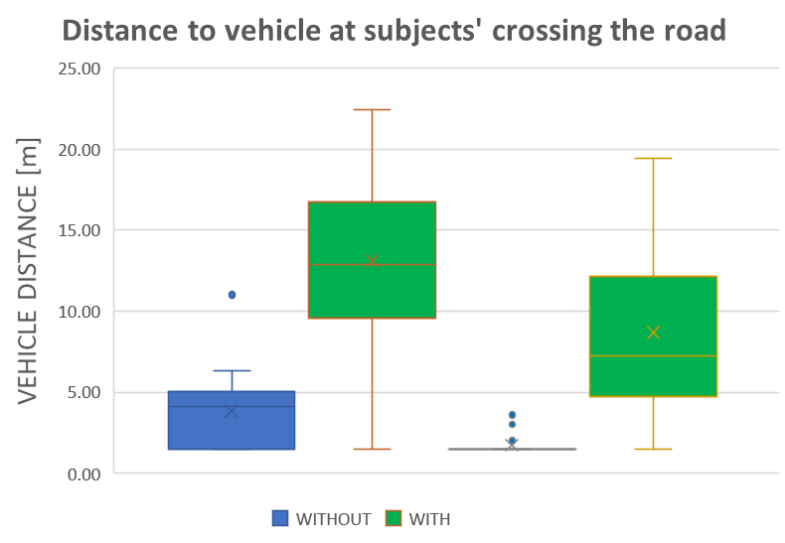

FiguRE 6. Situation recognition per vehicle distance.

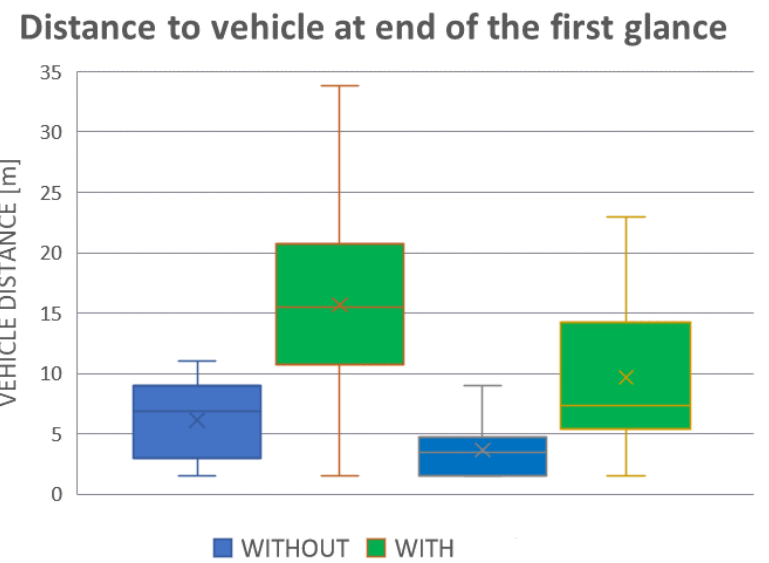

Figure 7. Subjects' decision making.

\subsection{Discussion}

An external vehicle communication provides subjects with helpful information to make the decision to cross the road sooner. This is proven by a decreased firstglance duration time that correlates to both the decrease in time from the first sight of the approaching vehicle and the increased distance to the vehicle when crossing the road. This effect results in a decrease of the mental load of a pedestrian when crossing the road. This was compared to one of the related naturalistic studies without the involvement of eHMI that analyses pedestrian's eye behaviour of subjects. Here, while performing the task, the subjects are to follow the approaching vehicle, driver's possible signals, and the surrounding area to assess the situation safety, distance to the approaching vehicle etc. [15]. The elimination of the human agent from the system is supposed to decrease the failure probability in systems involving both the humans and the machines [7]. The presence of a communication interface may thus replace the pedestrian to driver communication. Moreover, a standardized message of e-HMI will be more understandable to a broader group of people as compared to individual gestures provided by drivers.
The tested external communication concept focused on the information conveyed to subjects in one expected location, which also contributed to the shorter time needed for the risk mitigation in the different driving modes. According to the results, in this case, more subjects tend to cross the road both sooner and at a safer distance. The observed measures (first glance, decision taking time, i.e., the time from the end of the first glance to the start of walking and distance to collision) may be taken as valid measures for an assessment of external visualization concepts for a further verification of an external vehicle communication.

Trust towards autonomous vehicles corresponds to human awareness or familiarity with their concept and interface. This is confirmed by a related research, where the quantitative analyses of the study correlate with the decrease in decision time due to an external visualization interface presented [16]. In [17], it has been shown that a higher level of trust was displayed by subjects that have a prior knowledge of the concept and/or their possible external communication interfaces.

Inconsistences in subject's perception of the vehicle's speed (subjective self-rating) motivate authors to focus the further methodology development on a quantitative research, which would allow adjustments of the objective tool with the observation of subjects' visual behaviour assisted by qualitative and subjective studies.

\section{Conclusion}

This study represents an experimental research that contributes to the safety and reliability of autonomous driving by studying the efficiency of the humanmachine communication. Based on the human behaviour, it provides analyses for the design and improvement of external HMI, thus increasing the safety of vulnerable road users and contributing to traffic safety in general.

The proposed method can be adjusted to a variety of traffic situations. It is also applicable to numerous interface designs. The method was developed based on statistical data of accident analyses and theories of their prevention. With a bigger data sample to be collected in future research, the results can be correlated to these statistics.

The developed method is to be further applied for testing and assessment of various communication interfaces to validate a clear information presentation, interface placement on vehicle's body and the intuitiveness of the e-HMI interface.

\section{REFERENCES}

[1] Taxonomy and definitions for terms related to driving automation systems for on-road motor vehicles. J3016_201806, 2018, https://doi.org/10.4271/j3016_201806 
[2] P. Wintersberger, A.-K. Frison, A. Riener, L. N. Boyle. Towards a personalized trust model for highly automated driving. In B. Weyers, A. Dittmar (eds.), Mensch und Computer 2016 - Workshopband. 2016. https://doi.org/10.18420/muc2016-ws08-0008

[3] Police of the Czech Republic. Accident statistics. [2020-03], https://www.policie.cz/clanek/ statistika-nehodovosti-900835.aspx.

[4] D. Dey, J. Terken. Pedestrian interaction with vehicles: Roles of explicit and implicit communication. In AutomotiveUI '17: Proceedings of the 9th International Conference on Automotive User Interfaces and Interactive Vehicular Applications, pp. 109-113. 2017. https://doi.org/10.1145/3122986.3123009

[5] A. Quistberg, T. D. Koepsell, L. N. Boyle, et al. Pedestrian signalization and the risk of pedestrian-motor vehicle collisions in lima, peru. Accident Analysis \& Prevention 70:273-281, 2014. https://doi.org/10.1016/j.aap.2014.04.012.

[6] Transport Research Centre. Speed Management. OECD Publishing, Paris, 2017. ISBN 92-821-0377-3.

[7] J. Šachl. Analýza nehod v silničním provozu. České vysoké učení technické, Praha, 2010. ISBN 978-80-01-04638-8.

[8] M. Pernkopf, H. Tiesler-Wittig. Lighting for automated vehicles - discussion on ways forward, informal document GRE-79-35, 2018. [2020-03], https://www.unece.org/fileadmin/DAM/trans/doc/ 2018/wp29gre/GRE-79-35e.PDF.

[9] T. Lagstrom, V. M. Lundgren. AVIP - autonomous vehicles' interaction with pedestrians. Master of science thesis, Chalmers University of Technology, Department of Product- and Production Development, Gothenborg, Sweden, 2015. http://publications.lib.chalmers. se/records/fulltext/238401/238401.pdf.

[10] Overview: Mercedes-Benz F 015 luxury in motion. [2020-03], http://media.daimler.com/ marsMediaSite/en/instance/ko/
Overview-Mercedes-Benz-F-015-Luxury-in-Motion. xhtml?oid=9904624.

[11] A. Rasouli, J. Tsotsos. Autonomous vehicles that interact with pedestrians: A survey of theory and practice. IEEE Transactions on Intelligent Transportation Systems 21(3):900-918, 2020. https://doi.org/10.1109/TITS.2019.2901817.

[12] P. Bouchner. Interactive driving simulators - history, design and their utilization in area of HMI research. International journal of systems applications, engineering \& development 10:179-188, 2016. ISSN 2074-1308.

[13] P. Bouchner, S. Novotný. Car dynamics model design for interactive driving simulation use. In Recent Researches in Applied Informatics, pp. 285-289. WSEAS Press, Athens, 2011. ISBN 978-1-61804-034-3.

[14] P. Bouchner, S. Novotný. System with driving simulation device for HMI measurements. In WSEAS Transactions on Systems, pp. 287-293. WSEAS Press, Athens, 2005. ISBN 960-8457-29-7.

[15] D. Dey, F. Walker, M. Martens, J. Terken. Gaze patterns in pedestrian interaction with vehicles: towards effective design of external human- machine interfaces for automated vehicles. In AutomotiveUI '19: Proceedings of the 11th International Conference on Automotive User Interfaces and Interactive Vehicular Applications, pp. 369-378. 2019. https://doi.org/10.1145/3342197.3344523

[16] K. Holländer, A. Colley, C. Mai, et al. Investigating the influence of external car displays on pedestrians' crossing behavior in virtual reality. In Proceedings of the 21st International Conference on Human-Computer Interaction with Mobile Devices and Services (MobileHCI 2019), pp. 1-11. 2019. https://doi.org/10.1145/3338286.3340138

[17] M. Matthews, G. Chowdhary, E. Kieson. Intent communication between autonomous vehicles and pedestrians, 2017. CoRRabs/1708.07123, arXiv: 1708.07123 